\title{
Formal Concept Analysis of higher order
}

\author{
Ondrej Krídlo, Patrik Mihalčin, Stanislav Krajči and Lubomír Antoni \\ University of Pavol Jozef Šafárik, Košice, Slovakia*
}

\begin{abstract}
The second order formal context is a formal context such that its object and attribute sets are disjoint unions of object and attribute sets of external formal contexts. Every subset of object or attribute set will be evaluated from concept lattice of corresponding external formal context. The paper provides a method how to compute such second order formal concepts by using of bonds between external formal contexts or by using of heterogeneous formal contexts methods. Last part of the paper shows how this structure generalizes homogenic fuzzy formal context and its derivation operators.
\end{abstract}

\section{Motivation example}

Imagine the following situation as a motivation. Lets have a group of people that everybody knows each other (schoolmates, co-workers, etc.). All of them are going to travel somewhere together and you (as an organizer of the trip) would like to know their requirements for accommodation. Consider the following formal context. Set of objects represents a group of co-workers (Anna, Bob, Cyril, David, Erik). Set of attributes expresses their requirements (TV, Wellness, Closeness to a city center, Restaurant in a hotel). An example of such context is in the following table. Lets denote the following table as $\mathcal{P}$ as preferences.

\begin{tabular}{|l|c|c|c|c|}
\hline $\mathcal{P}$ & TV & W & Ce & R \\
\hline Anna & $\bullet$ & & $\bullet$ & $\bullet$ \\
\hline Bob & $\circ$ & $\circ$ & $\bullet$ & $\bullet$ \\
\hline Cyril & $\circ$ & $\bullet$ & $\bullet$ & $\circ$ \\
\hline David & $\bullet$ & $\bullet$ & $\circ$ & \\
\hline Erik & $\bullet$ & $\bullet$ & $\circ$ & $\bullet$ \\
\hline
\end{tabular}

A particular formal concept of the given context describes a set of co-workers such that these people together have a common requirements for accommodation.

In addition, there are another two formal contexts. The first one describes a friendship relation inside the group of such people (denoted as $\mathcal{F}$ ). The second one describes a situation about hotels and services they offer (denoted as $\mathcal{H}$ ).

* Partially supported by grant VEGA 1/0832/12 and APVV-0035-10.

(C) paper author(s), 2013. Published in Manuel Ojeda-Aciego, Jan Outrata (Eds.): CLA 2013, pp. 117-128, ISBN 978-2-7466-6566-8, Laboratory L3i, University of La Rochelle, 2013. Copying permitted only for private and academic purposes. 


\begin{tabular}{|l|c|c|c|c|c|}
\hline $\mathcal{F}$ & Anna & Bob & Cyril & David & Erik \\
\hline Anna & $\bullet$ & $\circ$ & $\circ$ & & \\
\hline Bob & $\circ$ & $\bullet$ & $\bullet$ & $\circ$ & $\circ$ \\
\hline Cyril & $\circ$ & $\bullet$ & $\bullet$ & $\bullet$ & $\bullet$ \\
\hline David & & $\circ$ & $\bullet$ & $\bullet$ & $\bullet$ \\
\hline Erik & & $\circ$ & $\bullet$ & $\bullet$ & $\bullet$ \\
\hline
\end{tabular}

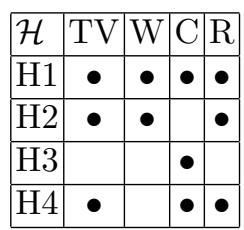

All contexts are filled by truth degrees from the following set $\{\bullet=$ true, $\circ=$ middle," " = false $\}$. Computing of $L$-concepts is based on Łukasiewicz logic.

Now, we aim at connecting such table data with known intercontextual mechanisms in order to obtain closed sets of friends from $\mathcal{F}$ that are able to stay in any of a closed set of hotels from $\mathcal{H}$. The hotels of this closed set offer as much requirements from $\mathcal{P}$ as it gets.

\section{Preliminaries}

\section{$2.1 \quad$ Basics}

Formal Concept Analysis (FCA) as an applied Lattice Theory [7] has become a very useful tool for discovering of hidden knowledge inside a data of objectattribute table, so called formal contexts. Fundamental construction of FCA is a Galois connection between complete lattices of all subsets of objects and attributes. A Galois connection consists of two mappings such that a composition of these mappings form a closure operators on each subsets of complete lattice. Pair of closed subset of objects and subset of attributes connected to each other by the Galois connection is called formal concept. The set of formal concepts forms a complete lattice. The mentioned notions were generalized over a fuzzy logic based on a complete residuated lattice. The notions of order, Galois connection and complete lattice were also generalized by Bělohlávek in [3-6].

Definition 1. Complete residuated lattice is an algebra $\langle L, \wedge, \vee, 0,1, \otimes, \rightarrow\rangle$, where

- $\langle L, \wedge, \vee, 0,1\rangle$ is a complete lattice with top 1 and bottom 0 ,

$-\langle L, \otimes, 1\rangle$ is a commutative monoid,

$-\langle\otimes, \rightarrow\rangle$ is an adjoint pair, i.e.

$$
a \otimes b \leq c \text { is equivalent to } a \leq b \rightarrow c
$$

for any $a, b, c \in L$.

Definition 2. $L$-fuzzy formal context $\mathcal{C}$ is a triple $\langle B, A, r\rangle$, where $r: B \times A \rightarrow$ $L$ is an $L$-fuzzy binary relation and $L$ is the complete residuated lattice.

Definition 3. Let $\langle B, A, r\rangle$ be an $L$-fuzzy formal context. Lets define a pair of derivation operators $\langle\uparrow, \downarrow\rangle$ of the form $\uparrow: L^{B} \longrightarrow L^{A}$ and $\downarrow: L^{A} \longrightarrow L^{B}$, where

$$
\begin{aligned}
& \uparrow(f)(a)=\bigwedge_{b \in B}(f(b) \rightarrow r(b, a)) \text { for any } f \in L^{B} \text { and } a \in A, \\
& \downarrow(g)(b)=\bigwedge_{a \in A}(g(a) \rightarrow r(b, a)) \text { for any } g \in L^{A} \text { and } b \in B .
\end{aligned}
$$


Lemma 1. Let $\langle\uparrow, \downarrow\rangle$ be a pair of derivation operators defined on an L-fuzzy formal context $\langle B, A, r\rangle$. A pair $\langle\uparrow, \downarrow\rangle$ forms a Galois connection between complete lattices of all L-sets of objects $L^{B}$ and attributes $L^{A}$.

Definition 4. Let $\mathcal{C}=\langle B, A, r\rangle$ be an $L$-fuzzy formal context. Formal concept is a pair of L-sets $\langle f, g\rangle \in L^{B} \times L^{A}$ such that $\uparrow(f)=g$ and $\downarrow(g)=f$. The set of all $L$-concepts of $\mathcal{C}$ will be denoted by $\mathrm{FCL}(\mathcal{C})$. Object or attribute part of any concept is called extent or intent. Sets of all extents or intents of $\mathcal{C}$ will be denoted as $\operatorname{Ext}(\mathcal{C})$ or $\operatorname{Int}(\mathcal{C})$, respectively.

\subsection{Bonds and Chu correspondences}

FCA provides the useful methods how to connect two formal contexts. A structure of the so called Chu correspondence was introduced by Mori $[13,14]$ that is very close to the notion of bond [7]. The notions of Chu correspondence and bond were extended into $L$-fuzzy Chu correspondence and $L$-bond in [8]. The corresponding notions are introduced now.

Definition 5. Let $\mathcal{C}_{i}=\left\langle B_{i}, A_{i}, r_{i}\right\rangle$ for $i \in\{1,2\}$ be two $L$-fuzzy formal contexts. Pair of L-multimappings $\varphi=\left\langle\varphi_{L}, \varphi_{R}\right\rangle$ such that

$-\varphi_{L}: B_{1} \longrightarrow \operatorname{Ext}\left(\mathcal{C}_{2}\right)$,

$-\varphi_{R}: A_{2} \longrightarrow \operatorname{Int}\left(\mathcal{C}_{1}\right)$,

where $\uparrow_{2}\left(\varphi_{L}\left(o_{1}\right)\right)\left(a_{2}\right)=\downarrow_{1}\left(\varphi_{R}\left(a_{2}\right)\right)\left(o_{1}\right)$ for any $\left(o_{1}, a_{2}\right) \in B_{1} \times A_{2}$, is said to be an $L$-Chu correspondence between $\mathcal{C}_{1}$ and $\mathcal{C}_{2}$. Set of all $L$-Chu correspondences between $L$-contexts $\mathcal{C}_{1}$ and $\mathcal{C}_{2}$ will be denoted by $L$-ChuCors $\left(\mathcal{C}_{1}, \mathcal{C}_{2}\right)$.

Definition 6. Let $\mathcal{C}_{i}=\left\langle B_{i}, A_{i}, r_{i}\right\rangle$ for $i \in\{1,2\}$ be two $L$-fuzzy formal contexts. L-multimapping $\beta: B_{1} \longrightarrow \operatorname{Int}\left(\mathcal{C}_{2}\right)$, such that $\beta^{\mathrm{t}}: A_{2} \longrightarrow \operatorname{Ext}\left(\mathcal{C}_{1}\right)$, where $\beta^{\mathrm{t}}\left(a_{2}\right)\left(o_{1}\right)=\beta\left(o_{1}\right)\left(a_{2}\right)$ for any $\left(o_{1}, a_{2}\right) \in B_{1} \times A_{2}$, is said to be an $L$-bond. Set of all $L$-bonds beyween $L$-contexts $\mathcal{C}_{1}$ and $\mathcal{C}_{2}$ will be denoted by $L$-Bonds $\left(\mathcal{C}_{1}, \mathcal{C}_{2}\right)$.

Lemma 2. Let $\mathcal{C}_{i}=\left\langle B_{i}, A_{i}, r_{i}\right\rangle$ for $i \in\{1,2\}$ be two $L$-fuzzy formal contexts. Each set $L$-Bonds $\left(\mathcal{C}_{1}, \mathcal{C}_{2}\right)$ and $L$-ChuCors $\left(\mathcal{C}_{1}, \mathcal{C}_{2}\right)$ forms a complete lattice and, moreover, there exists a dual isomorphism between them.

The dual isomorphism between bonds and Chu correspondences is based on the following construction. Consider two $L$-fuzzy formal contexts $\mathcal{C}_{i}=\left\langle B_{i}, A_{i}, r_{i}\right\rangle$ for $i \in\{1,2\}$ and let $\beta \in L$-Bonds $\left(\mathcal{C}_{1}, \mathcal{C}_{2}\right)$, then $\left\langle\varphi_{\beta L}, \varphi_{\beta R}\right\rangle$ such that for any $\left(o_{1}, a_{2}\right) \in B_{1} \times A_{2}$

$$
\varphi_{\beta L}\left(o_{1}\right)=\downarrow_{2}\left(\beta\left(o_{1}\right)\right) \text { and } \varphi_{\beta R}\left(a_{2}\right)=\uparrow_{1}\left(\beta^{\mathrm{t}}\left(a_{2}\right)\right)
$$

is an $L$-Chu correspondence from $L$-ChuCors $\left(\mathcal{C}_{1}, \mathcal{C}_{2}\right)$.

On the other hand, let $\varphi \in L$-ChuCors $\left(\mathcal{C}_{1}, \mathcal{C}_{2}\right)$. Then $\beta_{\varphi}$ defined as

$$
\beta_{\varphi}\left(o_{1}\right)\left(a_{2}\right)=\downarrow_{1}\left(\varphi_{R}\left(a_{2}\right)\right)\left(o_{1}\right)=\uparrow_{2}\left(\varphi_{L}\left(o_{1}\right)\right)\left(a_{2}\right)
$$

for any $\left(o_{1}, a_{2}\right) \in B_{1} \times A_{2}$ is an $L$-bond from $L$-Bonds $\left(\mathcal{C}_{1}, \mathcal{C}_{2}\right)$. 


\subsection{Categorical relationship to fuzzy Galois connection}

Categories ChuCors and $L$-ChuCors of classical or fuzzy formal contexts and classical or fuzzy Chu correspondences are described in $[9,13]$. Important categorical property of $*$-autonomism is also proved in mentioned papers. The continuation of categorical research in [10] resulted in a categorical equivalence of $L$-ChuCors and a category $L$-CLLOS of so called completely lattice $L$-ordered sets and monotone fuzzy Galois connections. Equivalence is proved by constructing of equivalence functor between these categories.

Definition 7. Lets define a functor $\Gamma: L$-ChuCors $\longrightarrow L$-CLLOS in the following way:

1. $\Gamma(\mathcal{C})=\langle\langle L$-FCL $(\mathcal{C}), \approx\rangle, \preceq\rangle$ for any $L$-context $\mathcal{C}$

2. $\Gamma(\varphi)=\left\langle\lambda_{L}^{\varphi}, \lambda_{R}^{\varphi}\right\rangle$ for any $\varphi \in L$-ChuCors $\left(\mathcal{C}_{1}, \mathcal{C}_{2}\right)$ such that $\lambda_{L}^{\varphi}: \operatorname{FCL}\left(\mathcal{C}_{1}\right) \longrightarrow$ $\operatorname{FCL}\left(\mathcal{C}_{2}\right)$ and $\lambda_{R}^{\varphi}: \operatorname{FCL}\left(\mathcal{C}_{2}\right) \longrightarrow \operatorname{FCL}\left(\mathcal{C}_{1}\right)$

$$
\begin{aligned}
& \lambda_{L}^{\varphi}\left(\left\langle f, \uparrow_{1}(f)\right\rangle\right)=\left\langle\downarrow_{2} \uparrow_{2}\left(\varphi_{L+}(f)\right), \uparrow_{2}\left(\varphi_{L+}(f)\right)\right\rangle \\
& \lambda_{R}^{\varphi}\left(\left\langle\downarrow_{2}(g), g\right\rangle\right)=\left\langle\downarrow_{1}\left(\varphi_{R+}(g)\right), \uparrow_{1} \downarrow_{1}\left(\varphi_{R+}(g)\right)\right\rangle
\end{aligned}
$$

for any two L-concepts $\left\langle f, \uparrow_{1}(f)\right\rangle \in \operatorname{FCL}\left(\mathcal{C}_{1}\right)$ and $\left\langle\downarrow_{2}(g), g\right\rangle \in \operatorname{FCL}\left(\mathcal{C}_{2}\right)$, where for any multifunction $\omega: X \longrightarrow L^{Y}$ is $\omega_{+}: L^{X} \longrightarrow L^{Y}$ defined as $\omega_{+}(f)(y)=\bigvee_{x \in X} f(x) \otimes \omega(x)(y)$ for any $f \in L^{X}$ and $y \in Y$.

In [10] is proved that $\Gamma$ is the equivalence functor. Hence, it holds for the particular two $L$-concepts that

$$
\left(\left\langle f, \uparrow_{1}(f)\right\rangle \preceq_{1} \lambda_{R}^{\varphi}\left(\left\langle\downarrow_{2}(g), g\right\rangle\right)\right)=\left(\lambda_{L}^{\varphi}\left(\left\langle f, \uparrow_{1}(f)\right\rangle\right) \preceq_{2}\left\langle\downarrow_{2}(g), g\right\rangle\right) .
$$

Lemma 3. Consider two $L$-contexts $\mathcal{C}_{i}=\left\langle B_{i}, A_{i}, r_{i}\right\rangle$ for $i \in\{1,2\}$ and let $\varphi \in L$-ChuCors $\left(\mathcal{C}_{1}, \mathcal{C}_{2}\right)$. A functor $\Gamma(\varphi)$ is a fuzzy Galois connection between $\left\langle\left\langle\operatorname{FCL}\left(\mathcal{C}_{1}\right), \approx_{1}\right\rangle, \preceq_{1}\right\rangle$ and $\left\langle\left\langle\operatorname{FCL}\left(\mathcal{C}_{2}^{*}\right), \approx_{2}\right\rangle, \preceq_{2}\right\rangle$ where $\mathcal{C}_{2}^{*}=\left\langle A_{2}, B_{2}, r_{2}^{\mathrm{t}}\right\rangle$.

Proof. Due to order reversing of dual $L$-context $\mathcal{C}_{2}^{*}$ for any two $L$-concepts we obtain $\left(\left\langle f, \uparrow_{1}(f)\right\rangle \preceq_{1} \lambda_{R}^{\varphi}\left(\left\langle\downarrow_{2}(g), g\right\rangle\right)\right)=\left(\left\langle\downarrow_{2}(g), g\right\rangle \preceq_{2} \lambda_{L}^{\varphi}\left(\left\langle f, \uparrow_{1}(f)\right\rangle\right)\right)$.

\section{Formal concept analysis of second order}

Once we have introduced preliminaries, the formal context of second order and the corresponding results are presented now in details.

Definition 8. Consider two non-empty index sets $I$ and $J$ and an L-fuzzy formal context $\left\langle\bigcup_{i \in I} B_{i}, \bigcup_{j \in J} A_{j}, r\right\rangle$, whereby

- $B_{i_{1}} \cap B_{i_{2}}=\emptyset$ for any $i_{1}, i_{2} \in I$,

- $A_{j_{1}} \cap A_{j_{2}}=\emptyset$ for any $j_{1}, j_{2} \in J$,

- $r: \bigcup_{i \in I} B_{i} \times \bigcup_{j \in J} A_{j} \longrightarrow L$. 
Moreover, consider two non-empty sets of L-contexts notated

- $\left\{\mathcal{C}_{i}=\left\langle B_{i}, T_{i}, p_{i}\right\rangle: i \in I\right\}$

- $\left\{\mathcal{D}_{j}=\left\langle O_{j}, A_{j}, q_{j}\right\rangle: j \in J\right\}$.

Formal context of second order is a tuple

$$
\left\langle\bigcup_{i \in I} B_{i},\left\{\mathcal{C}_{i} ; i \in I\right\}, \bigcup_{j \in J} A_{j},\left\{\mathcal{D}_{j} ; j \in J\right\}, \bigcup_{(i, j) \in I \times J} r_{i, j}\right\rangle,
$$

where $r_{i, j}: B_{i} \times A_{j} \longrightarrow L$ defined as $r_{i, j}(o, a)=r(o, a)$ for any $o \in B_{i}$ and $a \in A_{j}$.

In what follows, consider the below used notation. Lets have an $L$-set $f$ : $\prod_{i \in I} X_{i} \longrightarrow L$ for a non-empty universe set $X=\bigcup_{i \in I} X_{i}$, where $X_{i_{1}} \cap X_{i_{2}}=\emptyset$ for any $i_{1}, i_{2} \in I$. Then $f^{i}: X_{i} \longrightarrow L$ is defined as $f^{i}(x)=f(x)$ for an arbitrary $x \in X_{i}$ and $i \in I$.

With the help of functor $\Gamma$, we define the mappings between products of fuzzy concept lattices of objects and attributes formal contexts of the following form:

Definition 9. Lets define the mappings $\langle\Uparrow, \Downarrow\rangle$ as follows

$$
\begin{aligned}
\Uparrow: \prod_{i \in I} \mathrm{FCL}\left(\mathcal{C}_{i}\right) \longrightarrow \prod_{j \in J} \mathrm{FCL}\left(\mathcal{D}_{j}\right) \text { and } \Downarrow: \prod_{j \in J} \mathrm{FCL}\left(\mathcal{D}_{j}\right) \longrightarrow \prod_{i \in I} \mathrm{FCL}\left(\mathcal{C}_{i}\right) \\
\Uparrow(\Phi)^{j}=\bigwedge_{i \in I} \lambda_{i j L}\left(\Phi^{i}\right), \text { for any } \Phi \in \prod_{i \in I} \mathrm{FCL}\left(\mathcal{C}_{i}\right) \\
\Downarrow(\Psi)^{i}=\bigwedge_{j \in J} \lambda_{i j R}\left(\Psi^{j}\right), \text { for any } \Psi \in \prod_{j \in J} \operatorname{FCL}\left(\mathcal{D}_{j}\right)
\end{aligned}
$$

such that $\lambda_{i j}=\left\langle\lambda_{i j L}, \lambda_{i j R}\right\rangle=\Gamma\left(\varphi_{\rho_{i j}}\right)$, where

$$
\rho_{i j}=\bigvee\left\{\beta \in L-\operatorname{Bonds}\left(\mathcal{C}_{i}, \mathcal{D}_{j}\right):\left(\forall\left(o_{i}, a_{j}\right) \in B_{i} \times A_{j}\right) \beta\left(o_{i}\right)\left(a_{j}\right) \leq r_{i j}\left(o_{i}, a_{j}\right)\right\}
$$

Lemma 4. Let $\{\langle f, g\rangle\} \cup\left\{\left\langle f_{k}, g_{k}\right\rangle: k \in K\right\}$ be a non-empty set of L-concepts of any $L$-context and $K$ be a non-empty index set. Then

$$
\langle f, g\rangle \preceq \bigwedge_{k \in K}\left\langle f_{k}, g_{k}\right\rangle=\bigwedge_{k \in K}\left(\langle f, g\rangle \preceq\left\langle f_{k}, g_{k}\right\rangle\right) .
$$

Proof. Let the $L$-context be of the form $\langle B, A, r\rangle$. Hence

$$
\begin{aligned}
\langle f, g\rangle \preceq \bigwedge_{k \in K}\left\langle f_{k}, g_{k}\right\rangle & =\bigwedge_{o \in B}\left(f(o) \rightarrow\left(\bigwedge_{k \in K} f_{k}\right)(o)\right)=\bigwedge_{o \in B}\left(f(o) \rightarrow \bigwedge_{k \in K} f_{k}(o)\right) \\
& =\bigwedge_{k \in K} \bigwedge_{o \in B}\left(f(o) \rightarrow f_{k}(o)\right)=\bigwedge_{k \in K}\left(\langle f, g\rangle \preceq\left\langle f_{k}, g_{k}\right\rangle\right) .
\end{aligned}
$$


Lemma 5. Pair of mappings $\langle\Uparrow, \Downarrow\rangle$ forms a Galois connection between complete lattices $\left\langle\prod_{i \in I} \mathrm{FCL}\left(\mathcal{C}_{i}\right), \sqsubseteq_{I}\right\rangle$ and $\left\langle\prod_{j \in J} \mathrm{FCL}\left(\mathcal{D}_{j}\right), \sqsubseteq_{J}\right\rangle$.

Proof. Proof is provided in fuzzy ordering as a generalization of classical one.

$$
\begin{aligned}
\Psi \sqsubseteq J \Uparrow(\Phi) & =\bigwedge_{j \in J}\left(\Psi^{j} \preceq_{j} \Uparrow(\Phi)^{j}\right)=\bigwedge_{j \in J}\left(\Psi^{j} \preceq_{j} \bigwedge_{i \in I} \lambda_{i j L}\left(\Phi^{i}\right)\right) \\
& =\bigwedge_{i \in I} \bigwedge_{j \in J}\left(\Psi^{j} \preceq_{j} \lambda_{i j L}\left(\Phi^{i}\right)\right)=\bigwedge_{i \in I} \bigwedge_{j \in J}\left(\Phi^{i} \preceq_{i} \lambda_{i j R}\left(\Psi^{j}\right)\right) \\
& =\bigwedge_{i \in I}\left(\Phi^{i} \preceq_{i} \bigwedge_{j \in J} \lambda_{i j R}\left(\Psi^{j}\right)\right)=\bigwedge_{i \in I}\left(\Phi^{i} \preceq_{i} \Downarrow(\Psi)^{i}\right) \\
& =\Phi \sqsubseteq_{I} \Downarrow(\Psi) .
\end{aligned}
$$

\subsection{Simplification}

In this subsection will be presented a method that simplifies the previous consideration.

Definition 10. Let $\mathcal{C}_{i}=\left\langle B_{i}, A_{i}, r_{i}\right\rangle$ for $i \in\{1,2\}$ be two L-fuzzy contexts and let $\beta$ be an arbitrary $L$-bond between $\mathcal{C}_{1}$ and $\mathcal{C}_{2}$. Consider the following pair of mappings $\uparrow_{\beta}: L^{B_{1}} \longrightarrow L^{A_{2}}$ and $\downarrow_{\beta}: L^{A_{2}} \longrightarrow L^{B_{1}}$ such that

$$
\uparrow_{\beta}(f)(a)=\bigwedge_{o \in B_{1}}(f(o) \rightarrow \beta(o)(a)), \quad \downarrow_{\beta}(g)(o)=\bigwedge_{a \in A_{2}}(g(a) \rightarrow \beta(o)(a))
$$

for any $f \in L^{B_{1}}$ and $g \in L^{A_{2}}$.

Lemma 6. Let $\mathcal{C}_{i}=\left\langle B_{i}, A_{i}, r_{i}\right\rangle$ for $i \in\{1,2\}$ be two L-fuzzy contexts and let $\beta$ be an arbitrary $L$-bond between $\mathcal{C}_{1}$ and $\mathcal{C}_{2}$. A pair $\left\langle\uparrow_{\beta}, \downarrow_{\beta}\right\rangle$ forms a Galois connection between complete lattices $\left\langle\operatorname{Ext}\left(\mathcal{C}_{1}\right), \leq\right\rangle$ and $\left\langle\operatorname{Int}\left(\mathcal{C}_{2}\right), \leq\right\rangle$, where $\leq$ is ordering based on fuzzy sets inclusion.

Proof. Proof of the fact that $\left\langle\uparrow_{\beta}, \downarrow_{\beta}\right\rangle$ forms a Galois connection between $\left\langle L^{B_{1}}, \leq\right\rangle$ and $\left\langle L^{A_{2}}, \leq\right\rangle$ is simple, $\left\langle\uparrow_{\beta}, \downarrow_{\beta}\right\rangle$ is a pair of derivation operators for $L$-context $\left\langle B_{1}, A_{2}, \beta^{\mathrm{r}}\right\rangle$, where binary $L$-relation $\beta^{\mathrm{r}}$ is defined as $\beta^{\mathrm{r}}\left(o_{1}, a_{2}\right)=\beta\left(o_{1}\right)\left(a_{2}\right)$.

Now, we will show that $\left\langle\uparrow_{\beta}, \downarrow_{\beta}\right\rangle$ is a pair of mappings between complete lattices of extents and intents of $C_{1}$ and $C_{2}$, respectively. First, let $f$ be an extent of $C_{1}$.

$$
\begin{aligned}
\uparrow_{\beta}(f)(a) & =\bigwedge_{o \in B_{1}}(f(o) \rightarrow \beta(o)(a)) \\
& =\bigwedge_{o \in B_{1}}\left(f(o) \rightarrow \uparrow_{2}\left(\varphi_{\beta L}(o)\right)(a)\right) \\
& =\bigwedge_{o \in B_{1}}\left(f(o) \rightarrow \bigwedge_{b \in B_{2}}\left(\varphi_{\beta L}(o)(b) \rightarrow r_{2}(b, a)\right)\right)
\end{aligned}
$$




$$
\begin{aligned}
& =\bigwedge_{b \in B_{2}} \bigwedge_{o \in B_{1}}\left(f(o) \rightarrow\left(\varphi_{\beta L}(o)(b) \rightarrow r_{2}(b, a)\right)\right) \\
& \left.=\bigwedge_{b \in B_{2}} \bigwedge_{o \in B_{1}}\left(\left(\varphi_{\beta L}(o)(b) \otimes f(o)\right) \rightarrow r_{2}(b, a)\right)\right) \\
& \left.=\bigwedge_{b \in B_{2}}\left(\bigvee_{o \in B_{1}}\left(\varphi_{\beta L}(o)(b) \otimes f(o)\right) \rightarrow r_{2}(b, a)\right)\right) \\
& \left.=\bigwedge_{b \in B_{2}}\left(\varphi_{\beta L+}(f)(b) \rightarrow r_{2}(b, a)\right)\right) \\
& =\uparrow_{2}\left(\varphi_{\beta L+}(f)\right)(a) .
\end{aligned}
$$

So $\uparrow_{\beta}(f)$ is an intent of $C_{2}$.

Proof of $\downarrow_{\beta}(g)$ is an extent of $C_{1}$ is easy to obtain similarly with equality $\beta(o)(a)=\downarrow_{1}\left(\varphi_{\beta R}(o)\right)(a)$.

We define an $L$-context such that its $L$-concept lattice is isomorphic to a complete lattice of all second order formal concepts.

Definition 11. Let $\mathcal{K}$ be a second order formal context of the form

$$
\mathcal{K}=\left\langle\bigcup_{i \in I} B_{i},\left\{\mathcal{C}_{i}: i \in I\right\}, \bigcup_{j \in J} A_{j},\left\{\mathcal{D}_{j}: j \in J\right\}, \bigcup_{(i, j) \in I \times J} r_{i j}\right\rangle .
$$

Lets define an L-context $\widehat{\mathcal{K}}$

$$
\widehat{\mathcal{K}}=\left\langle\bigcup_{i \in I} B_{i}, \bigcup_{j \in J} A_{j}, \bigcup_{(i, j) \in I \times J} \rho_{i j}\right\rangle,
$$

where

$$
\rho_{i j}=\bigvee\left\{\beta \in L-\operatorname{Bonds}\left(\mathcal{C}_{i}, \mathcal{D}_{j}\right):\left(\forall\left(o_{i}, a_{j}\right) \in B_{i} \times A_{j}\right) \beta\left(o_{i}\right)\left(a_{j}\right) \leq r_{i j}\left(o_{i}, a_{j}\right)\right\} .
$$

Lemma 7. Concept lattices of $\mathcal{K}$ and $\widehat{\mathcal{K}}$ are isomorphic.

Proof. Let $\langle\Phi, \Psi\rangle$ be an $L$-concept of $\widehat{\mathcal{K}}$ and $o \in B_{i}$.

$$
\begin{aligned}
\begin{aligned}
\Phi^{i}(o)=\left(\downarrow_{\widehat{\mathcal{K}}}(\Psi)\right)^{i}(o) & =\bigwedge_{j \in J} \bigwedge_{a \in A_{j}}\left(\Psi^{j}(a) \rightarrow \rho_{i j}(o)(a)\right) \\
& =\bigwedge_{j \in J} \bigwedge_{a \in A_{j}}\left(\Psi^{j}(a) \rightarrow \downarrow_{i}\left(\varphi_{\rho_{i j} R}(a)\right)(o)\right) \\
& =\bigwedge_{j \in J} \downarrow_{i}\left(\varphi_{\rho_{i j} R+}\left(\Psi^{j}\right)\right)(o) .
\end{aligned} \\
\Phi^{i}=\left(\downarrow_{\widehat{\mathcal{K}}}(\Psi)\right)^{i}=\bigwedge_{j \in J} \downarrow_{i}\left(\varphi_{\rho_{i j} R+}\left(\Psi^{j}\right)\right)=\bigwedge_{j \in J} \operatorname{ext}\left(\lambda_{R}^{\varphi_{\rho_{i j}}}\left(\Psi^{j}\right)\right) \\
=\operatorname{ext}\left(\bigwedge_{j \in J} \lambda_{R}^{\left.\varphi_{\rho_{i j}}\left(\Psi^{j}\right)\right)=\operatorname{ext}\left(\Downarrow(\bar{\Psi})^{i}\right),}\right.
\end{aligned}
$$


where $\bar{\Psi}^{j}=\left\langle\downarrow_{j}\left(\Psi^{j}\right), \Psi^{j}\right\rangle$ for any $j \in J$. Then $\bar{\Phi}=\Downarrow(\bar{\Psi})$ and $\langle\bar{\Phi}, \bar{\Psi}\rangle$ is a second order concept of $\mathcal{K}$.

\section{Motivation example - solution}

The motivation example introduced in Section 1 can be considered as the second order formal context

$$
\langle\{\text { Anna,Bob,Cyril,David,Eva }\}, \mathcal{F},\{\mathrm{TV}, \mathrm{W}, \mathrm{Ce}, \mathrm{R}\}, \mathcal{H}, r\rangle,
$$

whereby $r$ represents the $L$-relation from $\mathcal{P}$. Firstly, we find a bond $\rho$ that is the closest to $r$.

\begin{tabular}{|l|c|c|c|c|}
\hline $\mathcal{P}$ & TV & W & Ce & R \\
\hline Anna & $\bullet$ & & $\bullet$ & $\bullet$ \\
\hline Bob & $\circ$ & $\circ$ & $\bullet$ & $\bullet$ \\
\hline Cyril & $\circ$ & $\bullet$ & $\bullet$ & $\circ$ \\
\hline David & $\bullet$ & $\bullet$ & $\circ$ & \\
\hline Erik & $\bullet$ & $\bullet$ & $\circ$ & $\bullet$ \\
\hline
\end{tabular}

\begin{tabular}{|l|c|c|c|c|c|}
\hline$\rho(\mathcal{P})$ & TV & W & Ce & $\mathrm{R}$ \\
\hline Anna & $\bullet$ & & $\bullet$ & $\bullet$ \\
\hline Bob & $\circ$ & $\circ$ & $\bullet$ & $\circ$ \\
\hline Cyril & $\circ$ & $\circ$ & $\bullet$ & $\circ$ \\
\hline David & & & $\circ$ & \\
\hline Erik & & & $\circ$ & \\
\hline
\end{tabular}

There are just six (instead of twenty-eight $L$-concepts of $\mathcal{P}$ ) $L$-concepts of $\rho(\mathcal{P})$ such that we can easily convert into the form of second order concepts. The following table contains the list of the all second order concepts.

\begin{tabular}{|c|c|}
\hline concepts of $\mathcal{F}$ & concepts of $\mathcal{H}$ \\
\hline$\{\circ / \mathrm{A}, \bullet / \mathrm{B}, \bullet / \mathrm{C}, \circ / \mathrm{D}, \circ / \mathrm{E}\}$ & $\{\mathrm{o} / \mathrm{TV}, \circ / \mathrm{W}, \bullet / \mathrm{Ce}, \circ / \mathrm{R}\}$ \\
$\{\circ / \mathrm{A}, \bullet / \mathrm{B}, \bullet / \mathrm{C}, \circ / \mathrm{D}, \circ / \mathrm{E}\}$ & $\{\bullet / \mathrm{H} 1, / \mathrm{H} 2, \circ / \mathrm{H} 3, \circ / \mathrm{H} 4\}$ \\
\hline$\{\bullet / \mathrm{A}, \bullet / \mathrm{B}, \bullet / \mathrm{C}, \bullet / \mathrm{D}, \bullet / \mathrm{E}\}$ & $\{/ \mathrm{TV}, / \mathrm{W}, \circ / \mathrm{C} e, / \mathrm{R}\}$ \\
$\{/ \mathrm{A}, \circ / \mathrm{B}, \circ / \mathrm{C}, / \mathrm{D}, / \mathrm{E}\}$ & $\{\bullet / \mathrm{H} 1, \circ / \mathrm{H} 2, \bullet / \mathrm{H} 3, \bullet / \mathrm{H} 4\}$ \\
\hline$\{\bullet / \mathrm{A}, \bullet / \mathrm{B}, \bullet / \mathrm{C}, \circ / \mathrm{D}, \circ / \mathrm{E}\}$ & $\{\mathrm{o} / \mathrm{TV}, / \mathrm{W}, \bullet / \mathrm{C} e, \circ / \mathrm{R}\}$ \\
$\{\mathrm{o} / \mathrm{A}, \circ / \mathrm{B}, \circ / \mathrm{C}, / \mathrm{D}, / \mathrm{E}\}$ & $\{\bullet / \mathrm{H} 1, / \mathrm{H} 2, \circ / \mathrm{H} 3, \bullet / \mathrm{H} 4\}$ \\
\hline$\{\bullet / \mathrm{A}, \circ / \mathrm{B}, \circ / \mathrm{C}, / \mathrm{D}, / \mathrm{E}\}$ & $\{\bullet / \mathrm{TV}, / \mathrm{W}, \bullet / \mathrm{C} e, \bullet / \mathrm{R}\}$ \\
$\{\bullet / \mathrm{A}, \circ / \mathrm{B}, \circ / \mathrm{C}, / \mathrm{D}, / \mathrm{E}\}$ & $\{\bullet / \mathrm{H} 1, / \mathrm{H} 2, / \mathrm{H} 3, \bullet / \mathrm{H} 4\}$ \\
\hline$\{\mathrm{o} / \mathrm{A}, \circ / \mathrm{B}, \circ / \mathrm{C}, / \mathrm{D}, / \mathrm{E}\}$ & $\{\bullet / \mathrm{TV}, \circ / \mathrm{W}, \bullet / \mathrm{C} e, \bullet / \mathrm{R}\}$ \\
$\{\bullet / \mathrm{A}, \bullet / \mathrm{B}, \bullet / \mathrm{C}, \circ / \mathrm{D}, \circ / \mathrm{E}\}$ & $\{\bullet / \mathrm{H} 1, / \mathrm{H} 2, / \mathrm{H} 3, \circ / \mathrm{H} 4\}$ \\
\hline$\{/ \mathrm{A}, \circ / \mathrm{B}, \circ / \mathrm{C}, / \mathrm{D}, / \mathrm{E}\}$ & $\{\bullet / \mathrm{TV}, \bullet / \mathrm{W}, \bullet / \mathrm{C} e, \bullet / \mathrm{R}\}$ \\
$\{\bullet / \mathrm{A}, \bullet / \mathrm{B}, \bullet / \mathrm{C}, \bullet / \mathrm{D}, \bullet / \mathrm{E}\}$ & $\{\bullet / \mathrm{H} 1, / \mathrm{H} 2, / \mathrm{H} 3, / \mathrm{H} 4\}$ \\
\hline
\end{tabular}

The first concept can be interpreted as follows. Friends Bob and Cyril with their common requirements should stay in hotel H1. They should stay also in $\mathrm{H} 3$ and $\mathrm{H} 4$ with a little relaxation of their requirements. The fourth concept is saying that Anna as a very lonely person should stay in $\mathrm{H} 1$ or H4. The second concept includes the whole group of co-workers who have very poor common requirements. Thus, all people should stay in an arbitrary hotel together. 


\section{Connection to heterogeneous formal contexts}

The fruitful idea is to view the second order formal context in terms of a heterogeneous formal context proposed in [2]. The corresponding notions of the underlying structures are introduced now.

Definition 12. Heterogeneous formal context is a tuple $\langle B, A, \mathcal{P}, R, \mathcal{U}, \mathcal{V}, \odot\rangle$, where

- $B$ and $A$ are non-empty sets,

$-\mathcal{P}=\left\{\left\langle P_{b, a}, \leq_{P_{b, a}}\right\rangle:(b, a) \in B \times A\right\}$ is a system of posets,

$-R$ is a mapping from $B \times A$ such that $R(b, a) \in P_{b, a}$ for any $b \in B$ and $a \in A$,

$-\mathcal{U}=\left\{\left\langle U_{b}, \leq_{U_{b}}\right\rangle: b \in B\right\}$ and $\mathcal{V}=\left\{\left\langle V_{a}, \leq_{V_{a}}\right\rangle: a \in A\right\}$ are systems of complete latices,

$-\odot=\left\{\mathrm{o}_{b, a}:(b, a) \in B \times A\right\}$ is a system of isotone and left-continuous mappings $\circ_{b, a}: U_{b} \times V_{a} \longrightarrow P_{b, a}$.

Lets describe our situation in terms of heterogeneous formal contexts. Below is the translation:

- $B$ and $A$ will be the index sets $I$ and $J$,

- complete lattices $U_{i}$ or $V_{j}$ for any $(i, j) \in B \times A=I \times J$ will be the complete lattices $\left\langle\operatorname{Ext}\left(\mathcal{C}_{i}\right), \leq\right\rangle$ and $\left\langle\operatorname{Int}\left(\mathcal{D}_{j}\right), \leq\right\rangle$,

- $P_{i, j}$ will be a complete lattice of all fuzzy relations from $L^{B_{i} \times A_{j}}$,

- any value of relation $r$ will be a binary relation $r(i, j)=r_{i, j} \in L^{B_{i} \times A_{j}}$,

- operation $\circ_{i, j}: \operatorname{Ext}\left(\mathcal{C}_{i}\right) \times \operatorname{Int}\left(\mathcal{D}_{j}\right) \longrightarrow L^{B_{i} \times A_{j}}$ is defined as

$$
\left(f \circ_{i, j} g\right)(b, a)=f(b) \otimes g(a)
$$

for any $f \in \operatorname{Ext}\left(\mathcal{C}_{i}\right)$ and $g \in \operatorname{Int}\left(\mathcal{D}_{j}\right)$ and any $(b, a) \in B_{i} \times A_{j}$. The mapping $\circ_{i, j}$ is isotone due to isotonicity of $\otimes$.

Lemma 8. The mapping $\circ_{i, j}$ is left-continuous.

Proof. Let

$$
\left(f_{k} \circ g\right)(b, a)=f_{k}(b) \otimes g(a) \leq m
$$

for all $k \in K$ and for some $(b, a) \in B \times A$ and $m \in L$. It is equivalent to inequality $f_{k}(b) \leq g(a) \rightarrow m$ for all $k \in K$. Hence, $\bigvee_{k \in K} f_{k}(b) \leq g(a) \rightarrow m$ and it is equivalent to

$$
\left(\bigvee_{k \in K} f_{k} \circ g\right)(b, a)=\bigvee_{k \in K} f_{k}(b) \otimes g(a) \leq m .
$$

Proof of left-continuity of the second argument is similar. 
Definition 13. Lets define a pair of derivation operators $\langle\nwarrow, \searrow\rangle$ of the following form $\nwarrow: \prod_{i \in I} \operatorname{Ext}\left(\mathcal{C}_{i}\right) \rightarrow \prod_{j \in J} \operatorname{Int}\left(\mathcal{D}_{j}\right)$ and $\searrow: \prod_{j \in J} \operatorname{Int}\left(\mathcal{D}_{j}\right) \rightarrow \prod_{i \in I} \operatorname{Ext}\left(\mathcal{C}_{i}\right)$ defined for heterogeneous formal context mentioned above as follows:

$$
\begin{aligned}
& \nwarrow(\Phi)^{j}=\bigvee\left\{g \in \operatorname{Int}\left(\mathcal{D}_{j}\right):(\forall i \in I) \Phi^{i} \circ_{i, j} g \leq r_{i, j}\right\} \\
& \searrow(\Psi)^{i}=\bigvee\left\{f \in \operatorname{Ext}\left(\mathcal{C}_{i}\right):(\forall j \in J) f \circ_{i, j} \Psi^{j} \leq r_{i, j}\right\}
\end{aligned}
$$

for any $\Phi \in \prod_{i \in I} \operatorname{Ext}\left(\mathcal{C}_{i}\right)$ and any $\Psi \in \prod_{j \in J} \operatorname{Int}\left(\mathcal{D}_{j}\right)$.

Lemma 9. Let $\mathcal{K}=\left\langle\bigcup_{i \in I} B_{i},\left\{C_{i} ; i \in I\right\}, \bigcup_{j \in J} A_{j},\left\{D_{j} ; j \in J\right\}, \bigcup_{(i, j) \in I \times J} r_{i, j}\right\rangle$ be a second order formal context. Then

$$
\uparrow_{\widehat{\mathcal{K}}}(\Phi) \leq \nwarrow(\Phi) \text { and } \downarrow_{\widehat{\mathcal{K}}}(\Psi) \leq \searrow(\Psi)
$$

for any $\Phi \in \prod_{i \in I} \operatorname{Ext}\left(\mathcal{C}_{i}\right)$ and $\Psi \in \prod_{j \in J} \operatorname{Int}\left(\mathcal{D}_{j}\right)$.

Proof. Let $j \in J$ be arbitrary.

$$
\begin{aligned}
\uparrow_{\widehat{\mathcal{K}}}(\Phi)^{j}(a) & =\bigwedge_{i \in I} \bigwedge_{o \in B_{i}}\left(\Phi^{i}(o) \rightarrow \rho_{i j}(o)(a)\right) \\
& =\bigvee\left\{g \in \operatorname{Int}\left(\mathcal{D}_{j}\right):(\forall i \in I)\left(\forall o \in B_{i}\right) \Phi^{i}(o) \otimes g(a) \leq \rho_{i j}(o)(a)\right\} .
\end{aligned}
$$

Then

$$
\begin{aligned}
& \uparrow_{\widehat{\mathcal{K}}}(\Phi)^{j}=\bigvee\left\{g \in \operatorname{Int}\left(\mathcal{D}_{j}\right):(\forall i \in I)\left(\forall o \in B_{i}\right) \Phi^{i} \circ_{i j} g \leq \rho_{i j}\right\} \\
& \text { because of } \rho_{i j} \leq r_{i j} \\
& \\
& \leq \bigvee\left\{g \in \operatorname{Int}\left(\mathcal{D}_{j}\right):(\forall i \in I)\left(\forall o \in B_{i}\right) \Phi^{i} \circ_{i j} g \leq r_{i j}\right\} \\
& \quad=\nwarrow(\Phi)^{j} .
\end{aligned}
$$

Hence $\uparrow_{\widehat{\mathcal{K}}}(\Phi) \leq \nwarrow(\Phi)$. Similarly for $\downarrow_{\widehat{\mathcal{K}}}$ and $\searrow$.

\section{Connections to standard homogenic fuzzy operators}

In this part, we focus on an appropriate generalization of the standard homogenic fuzzy formal concept derivation operators in two different ways.

\subsection{Singleton connection}

Lemma 10. Lets have an $L$-fuzzy formal context $\langle\{x\}, L, \lambda\rangle$, where for an arbitrary $k \in L$ is $\perp_{x}=\lambda(x, k)=k$. Any value $k \in L$ is an extent of $\perp_{x}$.

Proof. Let $k$ be an arbitrary value from $L$.

$$
\downarrow \uparrow(k)(x)=\bigwedge_{m \in L}(\uparrow(k)(m) \rightarrow m)=\bigwedge_{m \in L}\left(\bigwedge_{x \in\{x\}}(k \rightarrow m) \rightarrow m\right)
$$




$$
\begin{aligned}
& =\bigwedge_{m \in L: m \geq k}(1 \rightarrow m) \wedge \bigwedge_{m \in L: m<k}((k \rightarrow m) \rightarrow m) \\
& =\bigwedge_{m \in L: m \geq k} m \wedge \bigwedge_{m \in L: m<k}((k \rightarrow m) \rightarrow m)=\star \\
& \bigwedge_{\substack{m \in L: m \geq k \\
(k \rightarrow m) \rightarrow m}} m=k(L \text { is a complete lattice }) \\
& \bigwedge_{m \in L: m<k}((k \rightarrow m) \rightarrow m) \geq k \\
& \star=k
\end{aligned}
$$

So $\downarrow \uparrow(k)=k$ for any $k \in L$.

Lemma 11. Lets have an $L$-fuzzy formal context $\mathcal{C}=\langle B, A, r\rangle$ with derivation operators $\langle\uparrow, \downarrow\rangle$. Lets have a second order formal context

$$
\mathcal{K}=\left\langle\bigcup_{b \in B}\{b\},\left\{\perp_{b}: b \in B\right\}, \bigcup_{a \in A}\{a\},\left\{\perp_{a}^{*}: a \in A\right\}, \bigcup_{(b, a) \in B \times A} r(b, a)\right\rangle .
$$

Then concept lattices of $\mathcal{C}$ and $\mathcal{K}$ are isomorphic.

Proof. Let $\Phi \in \prod_{b \in B} \operatorname{Ext}\left(\perp_{b}\right)$. By previous lemma is easy to see that $\Phi \in L^{B}$. Moreover $r(b, a)$ for any $(b, a) \in B \times A$ as an arbitrary value from $L$ is an extent of $\perp_{b}$ and intent of $\perp_{a}^{*}=\left\langle L,\{a\},{ }^{\mathrm{t}} \lambda\right\rangle$. Hence any $r(b, a) \in L$-Bonds $\left(\perp_{b}, \perp_{a}^{*}\right)$.

$$
\uparrow_{\widehat{\mathcal{K}}}(\Phi)(a)=\bigwedge_{b \in B} \uparrow_{r(b, a)}(\Phi(o))=\bigwedge_{b \in B}(\Phi(o) \rightarrow r(o, a))=\uparrow(\Phi)(a) .
$$

Similarly for $\downarrow_{\widehat{\mathcal{K}}}=\downarrow$.

\section{$6.2 \neq$ connection}

Moreover, an another connection is presented in this subsection. The connection is based on the fact that concept lattice of $\langle X, X, \neq\rangle$ is isomorphic to $L^{X}$ in the case that $L$ is closed under double negation law.

Lemma 12. Lets have an $L$-fuzzy formal context $\mathcal{X}=\langle X, X, \neq\rangle$, where $L$ is closed under double negation law. Then any $L$-set from $L^{X}$ is closed in $\mathcal{X}$.

Proof. Lets have an arbitrary $L$-set $f \in L^{X}$.

$$
\begin{aligned}
\uparrow(f)(x) & =\bigwedge_{y \in X}(f(y) \rightarrow(y \neq x)) \\
& =\bigwedge_{y \in X: y \neq x}(f(y) \rightarrow 1) \wedge(f(x) \rightarrow(x \neq x)) \\
& =1 \wedge \neg f(x)=\neg f(x)
\end{aligned}
$$

Then $\downarrow \uparrow(f)(x)=\neg \neg f(x)=f(x)$. 
Lemma 13. Lets have an $L$-fuzzy formal context $\mathcal{C}=\langle B, A, r\rangle$, where $L$ is closed under double negation law. Concept lattice of $\mathcal{C}$ is isomorphic to a concept lattice of second order formal context $\mathcal{K}=\langle B, \mathcal{B}, A, \mathcal{A}, r\rangle$, where $\mathcal{B}=\langle B, B, \neq\rangle$ and $\mathcal{A}=\langle A, A, \neq\rangle$.

Proof. Index sets $I$ and $J$ are singletons in this case. So due to previous lemma $\Phi \in \operatorname{Ext}(\mathcal{B})=L^{B} . \Uparrow(\Phi)=\uparrow_{\rho}(f)$ where $\rho=\bigvee\{\beta \in L$-Bonds $(\mathcal{B}, \mathcal{A}): \beta \leq r\}$. Because of the previous lemma we know that any row and column or $r$ is closed in $\mathcal{B}$ and $\mathcal{A}$, respectively. Hence $r \in L$-Bonds $(\mathcal{B}, \mathcal{A})$ and $r=\rho$.

Finally $\uparrow_{\widehat{\mathcal{K}}}=\uparrow$. Similarly for $\downarrow_{\widehat{\mathcal{K}}}=\downarrow$.

\section{References}

1. L. Antoni, S. Krajči, O. Krídlo, B. Macek, and L. Pisková. Relationship between two FCA approaches on heterogeneous formal contexts. Proceedings of CLA, 93$102,2012$.

2. L. Antoni, S. Krajči, O. Krídlo, B. Macek, and L. Pisková. On heterogeneous formal contexts. Fuzzy Sets and Systems, In Press.

3. R. Bělohlávek. Fuzzy concepts and conceptual structures: induced similarities. Proceedings of Joint Conference on Information Sciences, pp. 179-182, 1998.

4. R. Bělohlávek. Lattices of fixed points of fuzzy Galois connections. Mathematical Logic Quartely, 47(1):111-116, 2001.

5. R. Bělohlávek. Concept lattices and order in fuzzy logic. Annals of Pure and Applied Logic, 128(1-3):277-298, 2004.

6. R. Bělohlávek. Lattice-type fuzzy order is uniquely given by its 1-cut: proof and consequences. Fuzzy Sets and Systems, 143:447-458, 2004.

7. B. Ganter and R. Wille. Formal concept analysis. Springer-Verlag, 1999.

8. O. Krídlo and M. Ojeda-Aciego. On the $L$-fuzzy generalization of Chu correspondences. International Journal of Computer Mathematics, 88(9):1808-1818, 2011.

9. O. Krídlo, S. Krajči, and M. Ojeda-Aciego. The category of $L$-Chu correspondences and the structure of $L$-bonds. Fundamenta Informaticae, 115(4):297-325, 2012.

10. O. Krídlo and M. Ojeda-Aciego. Linking $L$-Chu correspondences and completely lattice $L$-ordered sets. Proceedings of CLA, 233-244, 2012.

11. J. Medina and M. Ojeda-Aciego. Multi-adjoint t-concept lattices. Information Sciences, 180:712-725, 2010.

12. J. Medina and M. Ojeda-Aciego. On multi-adjoint concept lattices based on heterogeneous conjunctors. Fuzzy Sets and Systems, 208: 95-110, 2012.

13. H. Mori. Chu Correspondences. Hokkaido Matematical Journal, 37:147-214, 2008.

14. H. Mori. Functorial properties of Formal Concept Analysis. Proc ICCS, Lecture Notes in Computer Science, 4604:505-508, 2007.

15. J. Pócs. Note on generating fuzzy concept lattices via Galois connections. Information Sciences 185 (1):128-136, 2012.

16. J. Pócs. On possible generalization of fuzzy concept lattices. Information Sciences, 210: 89-98, 2012. 